Article

\title{
Symmetries and Reductions of Integrable Nonlocal Partial Differential Equations
}

\author{
Linyu Peng \\ Waseda Institute for Advanced Study, Waseda University, Tokyo 169-8050, Japan; 1.peng@aoni.waseda.jp \\ Received: 8 June 2019; Accepted: 2 July 2019; Published: 5 July 2019

\begin{abstract}
In this paper, symmetry analysis is extended to study nonlocal differential equations. In particular, two integrable nonlocal equations are investigated, the nonlocal nonlinear Schrödinger equation and the nonlocal modified Korteweg-de Vries equation. Based on general theory, Lie point symmetries are obtained and used to reduce these equations to nonlocal and local ordinary differential equations, separately; namely, one symmetry may allow reductions to both nonlocal and local equations, depending on how the invariant variables are chosen. For the nonlocal modified Korteweg-de Vries equation, analogously to the local situation, all reduced local equations are integrable. We also define complex transformations to connect nonlocal differential equations and differential-difference equations.
\end{abstract}

Keywords: continuous symmetry; symmetry reduction; integrable nonlocal partial differential equations

\section{Introduction}

Symmetry has proved to be fundamentally important in understanding the solutions of differential equations (see, e.g., [1-5]). It also reveals the integrability of partial differential equations (PDEs); for instance, the Ablowitz-Ramani-Segur conjecture stated that every ordinary differential equation (ODE) obtained by an exact reduction of an integrable evolution equation solvable by inverse scattering transforms is of the P-type, i.e., ODEs without movable critical points [6]. In this paper, powerful symmetry techniques are extended to study nonlocal differential equations with space and/or time reflections. This can not only provide insights for obtaining analytic solutions, but also reveal the integrability of the nonlocal equations. After writing down a general theory in Section 2, two integrable nonlocal differential equations-the nonlocal nonlinear Schrödinger (NLS) equation [7] and the nonlocal modified Korteweg-de Vries (mKdV) equation [8] —are separately investigated as illustrative examples. The results are immediately applicable to the many nonlocal differential equations proposed in the recent literature (see, e.g., [9-13].)

The nonlocal NLS equation

$$
\mathrm{i} q_{t}(x, t)+q_{x x}(x, t)+2 q^{2}(x, t) q^{*}(-x, t)=0,
$$

was derived by Ablowitz and Musslimani [7] by reduction of the AKNS system. The nonlocal NLS equation admits a great number of good properties that the classical NLS equation possesses, such as being PT-symmetric, admitting a Lax-pair and infinitely many conservation laws, and being solvable using inverse scattering transforms. Integrable nonlocal systems have recently received a great amount of attention with many newly-proposed models (e.g., the nonlocal vector NLS equation [13], a multi-dimensional extension of the nonlocal NLS equation [10], the nonlocal sine-Gordon equation, the nonlinear derivative NLS equation and related systems [9], the nonlocal $\mathrm{mKdV}$ equation [8], Alice-Bob physics [11], and the nonlocal Sasa-Satsuma equation [12], to mention only a few). Solutions of these systems have also been explored by many scholars; see, for example, [8,12,14-19]. 
One issue, as Ablowitz and Musslimani have noticed [7,9], is that reductions of nonlocal equations amount to nonlocal ODEs; for example, nonlocal Painlevé-type equations. In this paper, we show alternative ways which allow us to avoid such an inconvenience. We, first, classify all Lie point symmetries of the nonlocal NLS Equation (1) and the nonlocal mKdV equation [8]

$$
u_{t}(x, t)+u(x, t) u(-x,-t) u_{x}(x, t)+u_{x x x}(x, t)=0 .
$$

Then, possible symmetry reductions are conducted for both equations. We find that one may reduce a nonlocal differential equation to both nonlocal and local ODEs by choosing the invariant variables in different ways. In other words, we are able to kill all nonlocal terms in the reduced ODEs by choosing the invariant variables in a proper manner. In particular, for the nonlocal $\mathrm{mKdV}$ equation, all reduced local ODEs are integrable. These results are included in Sections 3 and 4. In Section 5, simple transformations are defined to connect nonlocal differential equations with differential-difference equations (DDEs).

\section{The Linearized Symmetry Condition for Nonlocal Differential Equations}

We, first, introduce the multi-index notations needed for the symmetry techniques of local differential equations (see, e.g., [5]), which will be extended to nonlocal differential equations.

Let $x=\left(x^{1}, x^{2}, \ldots, x^{m}\right) \in \mathbb{R}^{m}$ be the independent variables and let $u=\left(u^{1}, u^{2}, \ldots, u^{n}\right) \in \mathbb{R}^{n}$ be the dependent variables. Note that, in many occasions, people also tend to use $(x, t)$ to denote independent variables as the space $x$ and time $t$; this convention will occur in the next sections but, for now, we are happy without distinguishing one another. Partial derivatives of $u^{\alpha}$ are written in the multi-index form $u_{\mathbf{J}}^{\alpha}$, where $\mathbf{J}=\left(j_{1}, j_{2}, \ldots, j_{m}\right)$ with each index $j_{i}$ a non-negative integer, denoting the number of derivatives with respect to $x^{i}$; namely,

$$
u_{\mathbf{J}}^{\alpha}=\frac{\partial^{|\mathbf{J}|} u^{\alpha}}{\partial\left(x^{1}\right)^{j_{1}} \partial\left(x^{2}\right)^{j_{2}} \ldots \partial\left(x^{m}\right)^{j_{m}}},
$$

where $|J|=j_{1}+j_{2}+\cdots+j_{m}$. Consider a one-parameter group of Lie point transformations as follows:

$$
\widetilde{x}=\widetilde{x}(\varepsilon ; x, u), \quad \widetilde{u}=\widetilde{u}(\varepsilon ; x, u),
$$

subject to $\left.\widetilde{x}\right|_{\varepsilon=e}=x,\left.\widetilde{u}\right|_{\varepsilon=e}=u$. Here, $\varepsilon=e$ is the identity element of the one-parameter group. Define the total derivative with respect to $x^{i}$ as

$$
D_{i}=\frac{\partial}{\partial x^{i}}+\sum_{\alpha, J} u_{\mathbf{J}+\mathbf{1}_{i}}^{\alpha} \frac{\partial}{\partial u_{\mathbf{J}}^{\alpha}}
$$

where $\mathbf{1}_{i}$ is the $m$-tuple with only one non-zero entry 1 in the $i$-th position. The notation $\partial_{x^{i}}=\frac{\partial}{\partial x^{i}}$ and so forth will also be used. The corresponding infinitesimal generator is

$$
\mathbf{v}=\xi^{i}(x, u) \partial_{x^{i}}+\phi^{\alpha}(x, u) \partial_{u^{\alpha}}
$$

where

$$
\xi^{i}=\left.\frac{\mathrm{d} \widetilde{x}^{i}}{\mathrm{~d} \varepsilon}\right|_{\varepsilon=e^{\prime}} \quad \phi^{\alpha}=\left.\frac{\mathrm{d} \widetilde{u}^{\alpha}}{\mathrm{d} \varepsilon}\right|_{\varepsilon=e} .
$$

Note that the Einstein summation convention is used here (and elsewhere, if necessary). The prolonged generator $\operatorname{pr} \mathbf{v}$ can be written in terms of $u, \xi, \phi$, and their derivatives, as:

$$
\operatorname{pr} \mathbf{v}=\mathbf{v}+\sum_{\alpha,|\mathbf{J}| \geq 1} \phi_{\mathbf{J}}^{\alpha}(x,[u]) \partial_{u_{\mathbf{J}}^{\alpha}}
$$


where $[u]$ is shorthand for $u$ and finitely many of its partial derivatives, and the coefficients are recursively given by

$$
\phi_{\mathbf{J}+\mathbf{1}_{i}}^{\alpha}(x,[u])=D_{i} \phi_{\mathbf{J}}^{\alpha}(x,[u])-\left(D_{i} \xi^{j}(x, u)\right) u_{\mathbf{J}+\mathbf{1}_{j}}^{\alpha} .
$$

It is often more convenient to equivalently write prolonged generators in terms of the so-called characteristics of symmetries $Q^{\alpha}=\phi^{\alpha}-\xi^{i} u_{\mathbf{1}_{i}}^{\alpha}$; that is

$$
\operatorname{pr} \mathbf{v}=\xi^{i} D_{i}+\sum_{\alpha, \mathbf{J}}\left(D_{\mathbf{J}} Q^{\alpha}\right) \partial_{u_{\mathbf{J}}^{\alpha}} .
$$

Here, we use the shorthand notation $D_{\mathbf{J}}=D_{1}^{j_{1}} D_{2}^{j_{2}} \cdots D_{m}^{j_{m}}$ for $\mathbf{J}=\left(j_{1}, j_{2}, \ldots, j_{m}\right)$. The invariance of a system of local differential equations

$$
\left\{F_{k}(x,[u])=0\right\}_{k=1}^{l},
$$

corresponding to the transformations (4), leads to the linearized symmetry condition

$$
\operatorname{pr} \mathbf{v}\left(F_{k}(x,[u])\right)=0 \text {, whenever }\left\{F_{k}(x,[u])=0\right\}_{k=1}^{l}
$$

where $\mathbf{v}$ is the infinitesimal generator (6).

To extend the above analysis to the nonlocal equations of our interest, we define the following reflections for $i=1,2, \ldots, m$,

$$
\operatorname{Ref}^{i}:\left(x^{1}, \ldots, x^{i}, \ldots, x^{m}\right) \mapsto\left(x^{1}, \ldots,-x^{i}, \ldots, x^{m}\right)
$$

and

$$
\operatorname{Ref}^{i}: f\left(x^{1}, \ldots, x^{i}, \ldots, x^{m}\right) \mapsto f\left(x^{1}, \ldots,-x^{i}, \ldots, x^{m}\right),
$$

for a function $f$ defined on proper domains. In particular,

$$
\operatorname{Ref}^{i} u^{\alpha}\left(x^{1}, \ldots, x^{i}, \ldots, x^{m}\right)=u^{\alpha}\left(x^{1}, \ldots,-x^{i}, \ldots, x^{m}\right), \quad \alpha=1,2, \ldots, n .
$$

A system of nonlocal differential equations is, then, given by

$$
\mathcal{A}=\left\{F_{k}\left(x,[u],\left[\operatorname{Ref}^{i} u\right],\left[\operatorname{Ref}^{i} \circ \operatorname{Ref}^{j} u\right]_{i<j}, \ldots,[u(-x)]\right)=0\right\}_{k=1}^{l} .
$$

For simplicity, we will sometimes omit the arguments if they are local variables $x$. Let us consider transformations of the form (4) with the infinitesimal generator (6). Now, the prolongation formula involving the reflections becomes

$$
\begin{aligned}
\operatorname{pr}_{\operatorname{Ref}} \mathbf{v}= & \mathbf{v}+\sum_{\alpha,|\mathbf{J}| \geq 1} \phi_{\mathbf{J}}^{\alpha} \frac{\partial}{\partial u_{\mathbf{J}}^{\alpha}} \\
& +\sum_{i, \alpha,|\mathbf{J}| \geq 1}\left(\operatorname{Ref}^{i} \phi_{\mathbf{J}}^{\alpha}\right) \frac{\partial}{\partial\left(\operatorname{Ref}^{i} u_{\mathbf{J}}^{\alpha}\right)}+\sum_{i<j, \alpha,|\mathbf{J}| \geq 1}\left(\operatorname{Ref}^{i} \circ \operatorname{Ref}^{j} \phi_{\mathbf{J}}^{\alpha}\right) \frac{\partial}{\partial\left(\operatorname{Ref}^{i} \circ \operatorname{Ref}^{j} u_{\mathbf{J}}^{\alpha}\right)} \\
& +\cdots+\sum_{\alpha,|\mathbf{J}| \geq 1} \phi_{\mathbf{J}}^{\alpha}(-x,[u(-x)]) \frac{\partial}{\partial u_{\mathbf{J}}^{\alpha}(-x)},
\end{aligned}
$$

where the functions $\phi_{\mathbf{J}}^{\alpha}=\phi_{\mathbf{J}}^{\alpha}(x,[u])$ are again defined by (9). Invariance of the nonlocal system (16) with respect to the transformations (4) is equivalent to the linearized symmetry condition that

$$
\operatorname{pr}_{\operatorname{Ref}} \mathbf{v}\left(F_{k}\left(x,[u],\left[\operatorname{Ref}^{i} u\right],\left[\operatorname{Ref}^{i} \circ \operatorname{Ref}^{j} u\right]_{i<j}, \ldots,[u(-x)]\right)\right)=0, \text { whenever } \mathcal{A} \text { holds, }
$$


which are the first order terms about $\varepsilon$ in the Taylor expansions of the nonlocal system (16) evaluated at the new variables $\widetilde{x}, \widetilde{u}$, and so forth.

In the next two sections, we will apply this general theory to two integrable nonlocal differential equations: The nonlocal NLS equation and the nonlocal $\mathrm{mKdV}$ equation.

\section{The Nonlocal NLS Equation}

An integrable nonlocal NLS equation was proposed by Ablowitz and Musslimani [7]:

$$
\mathrm{i} q_{t}(x, t)+q_{x x}(x, t)+2 q^{2}(x, t) q^{*}(-x, t)=0,
$$

where $*$ denotes the complex conjugate and $q(x, t)$ is a complex-valued function of real variables $x$ and $t$. They showed that it possesses a Lax pair and infinitely many conservation laws, and is solvable by the inverse scattering transform. We study its continuous symmetries in this section.

\subsection{Lie Point Symmetries}

As $q(x, t)$ is complex-valued, two alternative approaches may be used to calculate its continuous symmetries. Under the co-ordinate $\left(x, t, q(x, t), q^{*}(x, t)\right)$, we consider the following local transformations

$$
\begin{aligned}
x & \mapsto x+\varepsilon \xi\left(x, t, q(x, t), q^{*}(x, t)\right)+O\left(\varepsilon^{2}\right), \\
t & \mapsto t+\varepsilon \tau\left(x, t, q(x, t), q^{*}(x, t)\right)+O\left(\varepsilon^{2}\right), \\
q(x, t) & \mapsto q(x, t)+\varepsilon \phi\left(x, t, q(x, t), q^{*}(x, t)\right)+O\left(\varepsilon^{2}\right) .
\end{aligned}
$$

Again, we omit the arguments if they are local variables $(x, t)$. The corresponding infinitesimal generator is

$$
\mathbf{v}=\xi\left(x, t, q, q^{*}\right) \partial_{x}+\tau\left(x, t, q, q^{*}\right) \partial_{t}+\phi\left(x, t, q, q^{*}\right) \partial_{q} .
$$

From Section 2, we know that the prolongation formula for an infinitesimal generator $\xi(x, t, u) \partial_{x}+$ $\tau(x, t, u) \partial_{t}+\phi(x, t, u) \partial_{u}$ of local differential equations is (see, also, [5,20])

$$
\xi D_{x}+\tau D_{t}+Q \partial_{u}+\left(D_{x} Q\right) \partial_{u_{x}}+\left(D_{t} Q\right) \partial_{u_{t}}+\cdots+\left(D_{x}^{k} D_{t}^{l} Q\right) \partial_{\left(D_{x}^{k} D_{t}^{l} u\right)}+\cdots,
$$

where the characteristic function $Q$ is $Q=\phi-\xi u_{x}-\tau u_{t}, D_{x}^{k}$ denotes $k$ times of total derivatives with respect to $x$ and $D_{t}^{l}$ denotes $l$ times of total derivatives with respect to $t$. For the nonlocal NLS equation, we then adopt the following prolongation formula:

$$
\begin{aligned}
\operatorname{pr}_{\operatorname{Ref}} \mathbf{v}= & \mathbf{v}+\phi^{*}\left(-x, t, q(-x, t), q^{*}(-x, t)\right) \partial_{q^{*}(-x, t)}+\left(D_{t} \phi-\left(D_{t} \xi\right) q_{x}-\left(D_{t} \tau\right) q_{t}\right) \partial_{q_{t}} \\
& +\left(D_{x}^{2} \phi-\left(D_{x}^{2} \xi\right) q_{x}-2\left(D_{x} \xi\right) q_{x x}-\left(D_{x}^{2} \tau\right) q_{t}-2\left(D_{x} \tau\right) q_{t x}\right) \partial_{q_{x x}}+\cdots
\end{aligned}
$$

It is generalised from the prolongation formula (22) for symmetries of local differential equations with real variables, adding the conjugate terms and their prolongations.

A vector field $\mathbf{v}$ generates a group of symmetries for the nonlocal NLS equation if the following linearized symmetry condition

$$
\operatorname{pr}_{\operatorname{Ref}} \mathbf{v}\left(\mathrm{i} q_{t}+q_{x x}+2 q^{2} q^{*}(-x, t)\right)=0
$$

holds identically for all solutions of the nonlocal NLS Equation (19). We, first, expand the left hand side of (24) and obtain

$$
\begin{aligned}
\mathrm{i}\left(D_{t} \phi-\left(D_{t} \xi\right) q_{x}-\left(D_{t} \tau\right) q_{t}\right)+D_{x}^{2} \phi & -\left(D_{x}^{2} \xi\right) q_{x}-2\left(D_{x} \xi\right) q_{x x}-\left(D_{x}^{2} \tau\right) q_{t}-2\left(D_{x} \tau\right) q_{t x} \\
& +4 q q^{*}(-x, t) \phi+2 q^{2} \phi^{*}\left(-x, t, q(-x, t), q^{*}(-x, t)\right)=0,
\end{aligned}
$$


restricted to solutions of the nonlocal NLS equation. We, then, substitute $q_{t}=\mathrm{i}\left(q_{x x}+2 q^{2} q^{*}(-x, t)\right)$ and $q_{t}^{*}=-\mathrm{i}\left(q_{x x}+2 q^{2} q^{*}(-x, t)\right)^{*}$, leading to a polynomial for $q_{x}, q_{x}^{*}, q_{x x}, q_{x x}^{*}$, and so forth, which equals zero identically. It is necessary and sufficient for the coefficients of the polynomial to vanish, amounting to a system of PDEs for $\xi, \tau$, and $\phi$, as follows:

$$
\begin{aligned}
& D_{x} \tau=0, \xi_{q}=0, \xi_{q^{*}}=0, \phi_{q^{*}}=0, \phi_{q q}=0, \tau_{t}-2 \xi_{x}=0, \mathrm{i} \xi_{t}+\xi_{x x}-2 \phi_{x q}=0, \\
& \mathrm{i} \phi_{t}+\phi_{x x}-2\left(\phi_{q}-\tau_{t}\right) q^{2} q^{*}(-x, t)+4 q q^{*}(-x, t) \phi+2 q^{2} \phi^{*}\left(-x, t, q(-x, t), q^{*}(-x, t)\right)=0 .
\end{aligned}
$$

The general solution of the above system is

$$
\xi=-C_{1} x+\mathrm{i} C_{2} t+C_{4}, \tau=-2 C_{1} t+C_{5}, \phi=\left(C_{1}+\mathrm{i} C_{3}-\frac{1}{2} C_{2} x\right) q,
$$

where $C_{1}, C_{2}$, and $C_{3}$ are real-valued, while $C_{4}$ and $C_{5}$ are complex-valued. Therefore, the symmetries of the nonlocal NLS equation are generated by the following five infinitesimal generators

$$
\partial_{x}, \quad \partial_{t}, \quad \mathrm{i} q \partial_{q}, \quad-x \partial_{x}-2 t \partial_{t}+q \partial_{q}, \quad \mathrm{i} t \partial_{x}-\frac{1}{2} x q \partial_{q} .
$$

They can, equivalently, be cast into evolutionary type (respectively), as follows

$$
\begin{gathered}
-q_{x} \partial_{q}, \quad \mathrm{i}\left(q_{x x}+2 q^{2} q^{*}(-x, t)\right) \partial_{q}, \quad \mathrm{i} q \partial_{q}, \\
\left(q+x q_{x}+2 \mathrm{i} t\left(q_{x x}+2 q^{2} q^{*}(-x, t)\right)\right) \partial_{q}, \quad\left(-\frac{1}{2} x q-\mathrm{i} t q_{x}\right) \partial_{q} .
\end{gathered}
$$

Alternatively, we can define $q(x, t)=u(x, t)-\mathrm{i} v(x, t)$, where $u(x, t)$ and $v(x, t)$ are real-valued functions, and use the symmetry prolongation formula for real-valued differential equations to calculate the symmetries. Now, the infinitesimal generator is

$$
\mathbf{v}=\xi(x, t, u, v) \partial_{x}+\tau(x, t, u, v) \partial_{t}+\phi(x, t, u, v) \partial_{u}+\eta(x, t, u, v) \partial_{v},
$$

and the equation becomes

$$
\left\{\begin{array}{l}
u_{t}-v_{x x}-4 u v u(-x, t)+2\left(u^{2}-v^{2}\right) v(-x, t)=0 \\
v_{t}+u_{x x}+4 u v v(-x, t)+2\left(u^{2}-v^{2}\right) u(-x, t)=0 .
\end{array}\right.
$$

The following symmetries are obtained for the system above, using the linearized symmetry condition (18) again:

$$
\partial_{x}, \partial_{t},-v \partial_{u}+u \partial_{v}, \quad-x \partial_{x}-2 t \partial_{t}+u \partial_{u}+v \partial_{v} .
$$

They correspond to the first four generators of (28). The last one obtained above does not appear here, since it will transform the real-valued $x$ to a complex-valued argument as $\xi=\mathrm{i} t$.

\subsection{Symmetry Reductions}

Next, we will use the symmetries to conduct possible reductions. We choose to use the symmetries (28) with complex variables. The simplest reduction one would expect is probably traveling-wave solutions, which are difficult to obtain here, as the invariant $x-a t$ becomes $-x-a t$ at $(-x, t)$.

Consider the most general infinitesimal generator

$$
a \partial_{x}+b \partial_{t}+c \mathrm{i} q \partial_{q}+d\left(-x \partial_{x}-2 t \partial_{t}+q \partial_{q}\right)+e\left(\mathrm{i} t \partial_{x}-\frac{1}{2} x q \partial_{q}\right)
$$


where $a, b, c, d$, and $e$ are arbitrary constants. The invariant variables can be found by solving the characteristic equations

$$
\frac{\mathrm{d} x}{a-d x+\mathrm{i} e t}=\frac{\mathrm{d} t}{b-2 d t}=\frac{\mathrm{d} q}{\mathrm{i} c q+d q-\frac{1}{2} e x q}
$$

and we summarize the results as follows. Note that the equation depends on $q(x, t)$ and $q^{*}(-x, t)$ simultaneously, and we must select the constants properly to make the invariants meaningful.

- If $d=b=0$ (and $a^{2}+e^{2} \neq 0$ ), we have

$$
\begin{aligned}
y & =t, \\
q(x, t) & =\exp \left\{\frac{x(4 \mathrm{i} c-e x)}{4(\mathrm{i} e t+a)}\right\} p(t) .
\end{aligned}
$$

When $a=0$ and $e \neq 0$, the reduced equation is

$$
\mathrm{i} e^{2} p^{\prime}(t)+\frac{\mathrm{ie}^{2}}{2 t} p(t)+\frac{c^{2}}{t^{2}} p(t)+2|p(t)|^{2} p(t)=0
$$

- If $d=0$ and $b \neq 0$, we have

$$
\begin{aligned}
y & =b x-\frac{1}{2} \mathrm{i} e t^{2}-a t \\
q(x, t) & =\exp \left\{-\frac{a e}{4 b^{2}} t^{2}-\frac{e}{2 b^{2}} t y+\mathrm{i}\left(\frac{c}{b} t-\frac{e^{2}}{12 b^{2}} t^{3}\right)\right\} p(y) .
\end{aligned}
$$

As $b \neq 0$, we must choose $a=e=0$. Next, we consider the corresponding reductions to nonlocal and local ODEs separately (here and throughout).

- Reduction to a nonlocal ODE. If we choose $y=x$ and $q(x, t)=\exp (\mathrm{i} c t) p(y)$, we obtain the nonlocal Painlevé-type equation as shown in [7]:

$$
p^{\prime \prime}(y)-c p(y)+2 p^{2}(y) p^{*}(-y)=0 .
$$

Note that, since $p(y)$ is invariant, and so is $p(-y)$; namely, the nonlocal invariant is

$$
p(-y)=\exp (-\mathrm{i} c t) q(-x, t)
$$

- Reduction to a local ODE. Alternatively, we may choose the invariants as $y=x^{2}$ and $q(x, t)=\exp (\mathrm{i} c t) p(y)$. The reduced equation is a local ODE

$$
4 y p^{\prime \prime}(y)=c p(y)-2 p^{\prime}(y)-2|p(y)|^{2} p(y) .
$$

If we assume that $p(y)$ is real, the solution of the above equation can be expressed, using the Jacobi elliptic function, as

$$
p(y)=C_{2} \sqrt{\frac{c}{C_{2}^{2}+c-1}} \operatorname{sn}\left(\sqrt{\frac{c}{C_{2}^{2}+c-1}}\left(\sqrt{-(c-1) y}+C_{1}\right), \frac{C_{2}}{\sqrt{c-1}}\right),
$$

where $C_{1}$ and $C_{2}$ are integration constants. The above equation can actually be written in a simpler form by introducing $y=z^{2}$ and $\widehat{p}(z)=p(y)$; the resulting equation is

$$
\hat{p}^{\prime \prime}(z)=c \widehat{p}(z)-2|\widehat{p}(z)|^{2} \widehat{p}(z) .
$$


- If $d \neq 0$, we have

$$
\begin{aligned}
y= & \frac{d^{2} x-a d+\mathrm{i} e(d t-b)}{d^{2} \sqrt{|2 d t-b|}}, \\
q(x, t)= & \exp \left\{\left(\frac{a e}{4 d^{2}}-\frac{1}{2}\right) \ln |2 d t-b|+\frac{e}{2 d} y \sqrt{|2 d t-b|}\right\} \times \\
& \quad \exp \left\{-\mathrm{i} \frac{e^{2}}{4 d^{2}} t+\mathrm{i}\left(\frac{b e^{3}}{8 d^{3}}-\frac{c}{2 d}\right) \ln |2 d t-b|\right\} p(y) .
\end{aligned}
$$

Now, we must set $a=e=0$.

- Reduction to a nonlocal ODE. Let

$$
\begin{aligned}
y & =\frac{x}{\sqrt{|2 d t-b|}} \\
q(x, t) & =\exp \left\{-\left(\frac{1}{2}+\frac{\mathrm{i} c}{2 d}\right) \ln |2 d t-b|\right\} p(y) .
\end{aligned}
$$

The reduced equation is a nonlocal ODE

$$
p^{\prime \prime}(y)=(\mathrm{i} d-c) p(y)+\mathrm{i} d y p^{\prime}(y)-2 p^{2}(y) p^{*}(-y) .
$$

- Reduction to a local ODE. If we choose the invariant variables by

$$
\begin{aligned}
y & =\frac{x^{2}}{2 d t-b^{\prime}} \\
q(x, t) & =\exp \left\{-\left(\frac{1}{2}+\frac{\mathrm{i} c}{2 d}\right) \ln |2 d t-b|\right\} p(y),
\end{aligned}
$$

the reduced equation is local; that is,

$$
4 y p^{\prime \prime}(y)=(\mathrm{i} d-c) p(y)+(2 \mathrm{i} d y-2) p^{\prime}(y)-2|p(y)|^{2} p(y) .
$$

Introducing $y=z^{2}$ and $\widehat{p}(z)=p(y)$ changes the equation to

$$
\widehat{p}^{\prime \prime}(z)=(\mathrm{i} d-c) \hat{p}(z)+\mathrm{i} d z \hat{p}(z)-2|\widehat{p}(z)|^{2} \widehat{p}(z) .
$$

\section{The Nonlocal mKdV Equation}

The nonlocal $\mathrm{mKdV}$ equation we consider in this paper is (see, for example, [8])

$$
u_{t}(x, t)+u(x, t) u(-x,-t) u_{x}(x, t)+u_{x x x}(x, t)=0 .
$$

Assuming that the infinitesimal generator reads

$$
\xi(x, t, u) \partial_{x}+\tau(x, t, u) \partial_{t}+\phi(x, t, u) \partial_{u}+\phi(-x,-t, u(-x,-t)) \partial_{u(-x,-t)},
$$

its prolongation can be obtained using (17). From a similar procedure for applying the linearized symmetry condition (18) to the nonlocal NLS equation above, a straightforward calculation gives the following infinitesimal generators for symmetries of the nonlocal $\mathrm{mKdV}$ equation:

$$
\partial_{x}, \partial_{t}, \quad-x \partial_{x}-3 t \partial_{t}+u \partial_{u}
$$


We follow the same approach as for the nonlocal NLS equation to search for symmetry reductions. The most general symmetry generator can be denoted by

$$
a \partial_{x}+b \partial_{t}+c\left(-x \partial_{x}-3 t \partial_{t}+u \partial_{u}\right),
$$

where $a, b$, and $c$ are arbitrary constants. The characteristic equations read

$$
\frac{\mathrm{d} x}{a-c x}=\frac{\mathrm{d} t}{b-3 c t}=\frac{\mathrm{d} u}{c u} .
$$

- When $c=0$, it corresponds to the traveling-wave case.

- Reduction to a nonlocal ODE. The corresponding invariants are

$$
y=b x-a t \text { and } v(y)=u(x, t) .
$$

The reduced equation is

$$
b^{3} v^{\prime \prime \prime}(y)+b v(y) v(-y) v^{\prime}(y)-a v^{\prime}(y)=0 .
$$

When $b=0$, we obtain a constant solution; when $b \neq 0$, without loss of generality, it can be chosen as $b=1$; namely

$$
v^{\prime \prime \prime}(y)+v(y) v(-y) v^{\prime}(y)-a v^{\prime}(y)=0 .
$$

In principle, it can be integrated once, as it admits a symmetry generated by $\partial_{y}$, but will involve the inverse of nonlocal functions. We will show some of its special solutions with the assumption $a>0$.

* Exponential solutions:

$$
v(y)=C_{1} \exp \left(C_{2} y\right) \text { subject to } C_{1}^{2}+C_{2}^{2}=a .
$$

* Soliton solutions:

$$
v(y)= \pm \frac{2 \sqrt{6 a}}{\exp (\sqrt{a} y)+\exp (-\sqrt{a} y)} .
$$

- Reduction to a local ODE. We may, alternatively, introduce the invariants in another way; namely, $y=(b x-a t)^{2}$ and $v(y)=u(x, t)$. Now, the reduced equation reads

$$
4 b^{3} y v^{\prime \prime \prime}(y)+6 b^{3} v^{\prime \prime}(y)+b v^{2}(y) v^{\prime}(y)-a v^{\prime}(y)=0,
$$

which can be integrated once:

$$
4 b^{3} y v^{\prime \prime}(y)+2 b^{3} v^{\prime}(y)+\frac{b}{3} v^{3}(y)-a v(y)+C_{1}=0 .
$$

This equation can be further simplified by introducing $y=z^{2}$ and $\widehat{v}(z)=v(y)$, amounting to

$$
b^{3} \widehat{v}^{\prime \prime}(z)+\frac{b}{3} \widehat{v}^{3}(z)-a \widehat{v}(z)+C_{1}=0 .
$$

The final equation is solvable by letting $\widehat{v}(z)=w(\widehat{v})$; the general solution is

$$
z+C_{3}= \pm \int_{0}^{\widehat{v}(z)} \frac{\sqrt{6} b^{3 / 2}}{\sqrt{-b s^{4}+6 a s^{2}-12 C_{1} s+6 C_{2} b^{3}}} \mathrm{~d} s
$$


where $C_{1}, C_{2}$, and $C_{3}$ are integration constants.

- If $c \neq 0$, the invariants are

$$
y=(c x-a)(3 c t-b)^{-1 / 3} \text { and } v(y)=(3 c t-b)^{1 / 3} u(x, t) .
$$

Now, we must set $a=b=0$; namely, reduction related to the generator $-x \partial_{x}-3 t \partial_{t}+u \partial_{u}$. The related invariants are $y=t^{-1 / 3} x$ and $v(y)=t^{1 / 3} u(x, t)$, and we obtain the reduced equation as a local ODE

$$
v^{\prime \prime \prime}(y)-v^{2}(y) v^{\prime}(y)-\frac{v(y)+y v^{\prime}(y)}{3}=0 .
$$

It can be integrated once to the second Painlevé equation

$$
v^{\prime \prime}(y)=\frac{1}{3} v^{3}(y)+\frac{1}{3} y v(y)+C .
$$

Now, we are able to conclude that all reduced local ODEs for the nonlocal $\mathrm{mKdV}$ equation are integrable, analogously to the local situation.

Remark 1. In [7], the authors pointed out that similarity reduction of the nonlocal NLS equation may lead to nonlocal ODEs. However, as shown by the two illustrative examples, such an inconvenience can be overcome by choosing the invariant variables (or functions) in a proper manner and the reduced ODEs become local.

\section{A Remark on Transformations Between Nonlocal Differential Equations and Differential-Difference Equations}

In [21], the authors introduced variable transformations to connect nonlocal and local integrable equations. For instance, the nonlocal NLS equation becomes a local NLS equation under the transformation

$$
x=\mathrm{i} \widehat{x}, t=-\widehat{t}, q(x, t)=\widehat{q}(\widehat{x}, \widehat{t}) .
$$

The nonlocal complex mKdV equation becomes the local (classical) complex mKdV equation under the transformation

$$
x=\mathrm{i} \widehat{x}, t=-\mathrm{i} \widehat{t}, u(x, t)=\widehat{u}(\widehat{x}, \widehat{t}) .
$$

In this section, we will show the relations between nonlocal differential equations and DDEs through variable transformations.

For the nonlocal NLS equation, we consider the following transformations

$$
x=\exp (\widehat{x}), t=\widehat{t}, q(x, t)=\widehat{q}(\widehat{x}, \widehat{t}),
$$

where the variable $\widehat{x}$ is imaginary, making $x$ imaginary too. Let us drop the hats (always) and the nonlocal NLS equation becomes a DDE

$$
\mathrm{i} q_{t}+\exp (-2 x)\left(q_{x x}-q_{x}\right)+2 q^{2} q^{*}(x+\mathrm{i} \pi, t)=0 .
$$

Let us introduce the following transformations

$$
x=\exp (\widehat{x}), \quad t=\exp (\widehat{t}), u(x, t)=\widehat{u}(\widehat{x}, \widehat{t}),
$$

where the variables $\widehat{x}$ and $\widehat{t}$ are both imaginary. The nonlocal $\mathrm{mKdV}$ equation becomes

$$
\exp (-t) u_{t}+\exp (-x) u u(x+\mathrm{i} \pi, t+\mathrm{i} \pi) u_{x}+\exp (-3 x)\left(u_{x x x}-3 u_{x x}+2 u_{x}\right)=0 .
$$


Under the transformation $y=\exp (\widehat{y}), v(y)=\widehat{v}(\widehat{y})$, the reduced Equation (55) becomes

$$
b^{3} \exp (-2 y)\left(v^{\prime \prime \prime}(y)-3 v^{\prime \prime}(y)+2 v^{\prime}(y)\right)+(b v(y) v(y+\mathrm{i} \pi)-a) v^{\prime}(y)=0 .
$$

These DDEs can further be re-scaled and normalized. For example, taking $y=\mathrm{i} \pi \hat{y}$ and $v(y)=$ $\widehat{v}(\widehat{y})$, Equation (72) becomes

$$
b^{3} \exp (-\mathrm{i} 2 \pi y)\left(-\frac{1}{\pi^{2}} v^{\prime \prime \prime}(y)+\frac{3 \mathrm{i}}{\pi} v^{\prime \prime}(y)+2 v^{\prime}(y)\right)+(b v(y) v(y+1)-a) v^{\prime}(y)=0 .
$$

Similar DDEs were investigated in [22], but the variables were real-valued therein. In the same manner, the above DDEs transformed from the nonlocal NLS and $\mathrm{mKdV}$ equations can also be re-scaled, respectively, as follows:

$$
\mathrm{i} q_{t}+\exp (-\mathrm{i} 2 \pi x)\left(-\frac{1}{\pi^{2}} q_{x x}+\frac{\mathrm{i}}{\pi} q_{x}\right)+2 q^{2} q^{*}(x+1, t)=0,
$$

and

$$
\exp (-\mathrm{i} \pi t) u_{t}+\exp (-\mathrm{i} \pi x) u u(x+1, t+1) u_{x}+\exp (-\mathrm{i} 3 \pi x)\left(-\frac{1}{\pi^{2}} u_{x x x}+\frac{3 \mathrm{i}}{\pi} u_{x x}+2 u_{x}\right)=0 .
$$

Remark 2. The above examples show that simple transformations allow us to transfer nonlocal equations to DDEs. Apparently, similar transformations can be immediately introduced for other nonlocal differential equations/systems using the same manner.

\section{Conclusions}

In this paper, symmetry analysis was extended to study nonlocal differential equations. The general theory presented in Section 2 is applicable to any nonlocal differential equations involving space and/or time reflections. In particular, two integrable nonlocal equations-the nonlocal NLS equation and the nonlocal $\mathrm{mKdV}$ equation-served as illustrative examples. All Lie point symmetries of these two nonlocal PDEs were obtained and possible symmetry reductions to nonlocal and local ODEs were conducted. It was shown that, at least for the two illustrative examples, one can always carefully choose the invariant variables to ensure that all reduced differential equations are local.

Finally, we introduced some local transformations which transfer nonlocal differential equations to DDEs; there is potential, hence, to extend the existing theory for DDEs to nonlocal differential equations; for instance, the symmetries, conservation laws, and integrability of DDEs (see, for example, [22-27]). We will explore more in this direction in a separate project.

Funding: This work was partially supported by JSPS Grant-in-Aid for Scientific Research (No. 16KT0024), the MEXT 'Top Global University Project', and Waseda University Grant for Special Research Projects (Nos. 2019C-179, 2019E-036, 2019R-081).

Conflicts of Interest: The author declares no conflict of interest.

\section{References}

1. Ackerman, M.; Hermann, R. Sophus Lie's 1880 Transformation Group Paper; Math. Sci. Press: Brookline, MA, USA, 1975.

2. Bluman, G.W.; Cole, J.D. General similarity solution of the heat equation. J. Math. Mech. 1969, 18, $1025-1042$.

3. Bluman, G.W.; Kumei, S. Symmetries and Differential Equations; Springer: New York, NY, USA, 1989.

4. Hydon, P.E. Symmetry Methods for Differential Equations: A Beginner's Guide; Cambridge University Press: Cambridge, UK, 2000.

5. $\quad$ Olver, P.J. Applications of Lie Groups to Differential Equations, 2nd ed.; Springer: New York, NY, USA, 1993. 
6. Ablowitz, M.J.; Ramani, A.; Segur, H. Nonlinear evolution equations and ordinary differential equations of Painlevé type. Lett. Nuovo Cimento 1978, 23, 333-338. [CrossRef]

7. Ablowitz, M.J.; Musslimani, Z.H. Integrable nonlocal nonlinear Schrödinger equation. Phys. Rev. Lett. 2013, 110, 064105. [CrossRef] [PubMed]

8. Ji, J.L.; Zhu, Z.N. On a nonlocal modified Korteweg-de Vries equation: Integrability, Darboux transformation and soliton solutions. Commun. Nonlinear Sci. Numer. Simul. 2017, 42, 699-708. [CrossRef]

9. Ablowitz, M.J.; Musslimani, Z.H. Integrable nonlocal nonlinear equations. Stud. Appl. Math. 2017, 139, 7-59. [CrossRef]

10. Fokas, A.S. Integrable multidimensional versions of the nonlocal nonlinear Schrödinger equation. Nonlinearity 2016, 29, 319-324. [CrossRef]

11. Lou, S.Y.; Huang, F. Alice-Bob physics: Coherent solutions of nonlocal KdV systems. Sci. Rep. 2017, 7, 869. [CrossRef]

12. Song, C.Q.; Xiao, D.M.; Zhu, Z.N. Reverse space-time nonlocal Sasa-Satsuma equation and its solutions. J. Phys. Soc. Jpn. 2017, 86, 054001. [CrossRef]

13. Yan, Z. Integrable $\mathcal{P} \mathcal{T}$-symmetric local and nonlocal vector nonlinear Schrödinger equations: A unified two-parameter model. Appl. Math. Lett. 2015, 47, 61-68. [CrossRef]

14. Ablowitz, M.J.; Musslimani, Z.H. Inverse scattering transform for the integrable nonlocal nonlinear Schrödinger equation. Nonlinearity 2016, 29, 915-946. [CrossRef]

15. Gurses, M.; Pekcan, A. Nonlocal nonlinear Schrödinger equations and their soliton solutions. J. Math. Phys. 2018, 59, 051501. [CrossRef]

16. Khare, A.; Saxena, A. Periodic and hyperbolic soliton solutions of a number of nonlocal nonlinear equations. J. Math. Phys. 2015, 56, 032104. [CrossRef]

17. Song, C.Q.; Xiao, D.M.; Zhu, Z.N. Solitons and dynamics for a general integrable nonlocal coupled nonlinear Schrödinger equation. Commun. Nonlinear Sci. Numer. Simul. 2017, 45, 13-28. [CrossRef]

18. Xu, Z.X.; Chow, K.W. Breathers and rogue waves for a third order nonlocal partial differential equation by a bilinear transformation. Appl. Math. Lett. 2016, 56, 72-77. [CrossRef]

19. Zhou, Z.X. Darboux transformations and global solutions for a nonlocal derivative nonlinear Schrödinger equation. Commun. Nonlinear Sci. Numer. Simul. 2018, 62, 480-488. [CrossRef]

20. Kumei, S. Group theoretic aspects of conservation laws of nonlinear dispersive waves: KdV type equations and nonlinear Schrödinger equations. J. Math. Phys. 1977, 18, 256-264. [CrossRef]

21. Yang, B.; Yang, J. Transformations between nonlocal and local integrable equations. Stud. Appl. Math. 2017, 140, 178-201. [CrossRef]

22. Quispel, G.R.W.; Capel, H.W.; Sahadevan, R. Continuous symmetries of differential-difference equations: The Kac-van Moerbeke equation and Painlevé reduction. Phys. Lett. A 1992, 170, 379-383. [CrossRef]

23. Kupershmidt, B.A. Discrete Lax Equations and Differential-Difference Calculus; Astérisque: Paris, France, 1985.

24. Levi, D.; Winternitz, P.; Yamilov, R.I. Lie point symmetries of differential-difference equations. J. Phys. A Math. Theor. 2010, 43, 292002. [CrossRef]

25. Mikhailov, A.V.; Wang, J.P.; Xenitidis, P. Cosymmetries and Nijenhuis recursion operators for difference equations. Nonlinearity 2011, 24, 2079-2097. [CrossRef]

26. Peng, L. Symmetries, conservation laws, and Noether's theorem for differential-difference equations. Stud. Appl. Math. 2017, 139, 457-502. [CrossRef]

27. Yamilov, R. Symmetries as integrability criteria for differential difference equations. J. Phys. A Math. Gen. 2006, 39, R541-R623. [CrossRef]

(C) 2019 by the authors. Licensee MDPI, Basel, Switzerland. This article is an open access article distributed under the terms and conditions of the Creative Commons Attribution (CC BY) license (http:/ / creativecommons.org/licenses/by/4.0/). 